1 Universidade Federal dos Vales do Jequitinhonha e Mucuri (UFVJM) Diamantina (MG), Brasil. fabiana.paula@ufvjm. edu.br

2 Universidade Federal dos Vales do Jequitinhonha e Mucuri (UFVJM) Diamantina (MG), Brasil. cristhiane_carvalhais@ hotmail.com

3 Universidade Federal dos Vales do Jequitinhonha e Mucuri (UFVJM) Diamantina (MG), Brasil. delbafonseca@yahoo. com.br

${ }^{4}$ Fundação Oswaldo Cruz (Fiocruz), Instituto René Rachou - Belo Horizonte (MG), Brasil.

oamfilho@cpqr.fiocruz.br

5 Universidade Federal dos Vales do Jequitinhonha e Mucuri (UFVJM) Diamantina (MG), Brasil. renata.aline.andrade@ gmail.com

\section{Avaliação da atenção à saúde do adulto em um município-polo do Vale do Jequitinhonha (MG)}

\author{
Evaluation of adult health care in a city pole of Vale do Jequitinhonha \\ $(M G)$
}

Fabiana Angélica de Paula', Cristhiane Carvalhais Reis Silva², Delba Fonseca Santos $\mathbf{3}$, Olindo Assis Martins-Filho ${ }^{\mathbf{4}}$, Renata Aline de Andrade ${ }^{\mathbf{5}}$

RESUMO A qualidade da Atenção Primária à Saúde é avaliada a partir da presença e extensão dos atributos essenciais e derivados. O presente estudo avaliou esses atributos (saúde do adulto) na Estratégia Saúde da Família de um município do Vale do Jequitinhonha (MG). Os dados foram coletados por meio do PCATool. Foi observado escore geral abaixo do valor considerado satisfatório $(\geq 6,6)$, e escores satisfatórios foram identificados para apenas dois atributos. Percebeu-se uma melhor orientação para os atributos em apenas uma área de abrangência. Acredita-se que este trabalho poderá subsidiar futuras práticas e decisões acerca do investimento público, visando ao fortalecimento da atenção primária pelos gestores.

PALAVRAS-CHAVE Avaliação em saúde; Atenção Primária à Saúde; Estratégia Saúde da Família.

ABSTRACT The quality of Primary Health Care is evaluated from the presence and the extent of the essential and derived attributes. This current study has evaluated those attributes (adults' health) in the Family Health Strategy of a municipality located in the Vale of Jequitinhonha (MG). Data were harvested by PCAtool. It was observed general score below the value considered satisfactory $(\geq 6,6)$ and satisfying scores were identified for only two attributes: It was noticed a better orientation for the attributes in only one area of coverage. It is believed that this work may subsidize future practices and decisions about public investment, aiming at the strengthening of the Primary Care by municipal managers.

KEYWORDS Health evaluation; Primary Health Care; Family Health Strategy. 


\section{Introdução}

Em 2006, o Ministério da Saúde (MS) define a saúde da família como estratégia prioritária para a organização da Atenção Primária à Saúde (APS), levando em consideração os preceitos do Sistema Único de Saúde (SUS) (BRASIL, 2006). A Estratégia Saúde da Família (ESF) é entendida como uma estratégia de reorientação do modelo assistencial, operacionalizada mediante a implantação de equipes multiprofissionais em unidades básicas de saúde (BRASIL, 2007).

O Brasil, em decorrência do envelhecimento populacional, está enfrentando progressivamente a substituição das doenças infecciosas e transmissíveis por doenças crônicas e degenerativas. Neste sentido, a saúde do adulto torna-se ainda mais importante, sendo necessário questionar sobre o momento em que se deve trabalhar com a prevenção de agravos da saúde.

Diante do aumento da cobertura populacional da ESF e de seus serviços, cresce também a necessidade de produzir conhecimento científico sobre a APS, sendo a avaliação dos serviços de saúde um instrumento importante desse conhecimento (HARZHEIM ET AL., 2005). Desde 1998, o MS vem desenvolvendo ações de avaliação e monitoramento da APS em conjunto com as secretarias estaduais e municipais de saúde e com instituições de pesquisa, mediante o Pacto pela Atenção Básica, além dos estudos de linhas de base e, mais recentemente, da Avaliação para Melhoria da Qualidade (AMQ) da Estratégia Saúde da Família (FIGUEIREDO, 2013).

Apesar do esforço para o desenvolvimento de instrumentos de aplicabilidade nacional, observa-se que a abrangência dessas avaliações, inclusive da AMQ, ainda é limitada. Reflete-se, assim, a não institucionalização da cultura da avaliação, cujas causas podem estar relacionadas a duas ordens de dificuldades: seja para se chegar a um consenso sobre a pertinência dos resultados produzidos, seja para se utilizar desses mesmos resultados nos processos decisórios (BRASIL, 2010B) ou pela existência de desafios substanciais para estabelecer parâmetros de qualidade da Atenção Básica que sejam mensuráveis (RIBEIRO, 2009).

O conceito da APS foi sistematizado por Bárbara Starfield (2002) através de atributos denominados essenciais: acesso de primeiro contato, que é a acessibilidade e o uso do serviço a cada novo problema ou acompanhamento rotineiro de saúde; a longitudinalidade, que é o aporte regular de cuidados pela equipe de saúde ao longo do tempo; a integralidade que implica a prestação de um conjunto de serviços que atendam às necessidades da população; e a coordenação, que é a capacidade de garantir a continuidade da atenção no interior da rede de serviços. A autora também define três atributos derivados: a orientação familiar, consideração da família como o sujeito da atenção; a orientação comunitária, que procede do reconhecimento das necessidades familiares em função do contexto social; e a competência cultural, que envolve a atenção às necessidades de uma população com características culturais especiais.

Com base nessa definição, Starfield e pesquisadores (2002) produziram o instrumento Primary Care Assessment Tool (PCATool), um instrumento de avaliação da atenção primária voltada para a saúde da criança e um instrumento para a atenção primária voltada para adultos (STARFIELD, 2002; SHI; STARFIELD; XU, 2001).

Ao longo dos anos, essa ferramenta foi utilizada e validada em diversos países, como Espanha e Canadá (PASARín ET AL., 2007). No Brasil, estudos utilizando o PCATool demonstraram que a ESF teve impacto positivo sobre a mortalidade infantil, sobre a redução de internações por condições sensíveis à APS, sobre a qualidade da atenção pré-natal e de puericultura, assim como ampliação do acesso a serviços de saúde para população vulnerável do ponto de vista sociossanitário (HARZHEIM, 2013). 
Considera-se que os processos de avaliação da satisfação dos usuários nos serviços públicos de saúde podem atuar para a construção de uma nova perspectiva do cuidado e que, além de monitorar as atividades desses serviços, pode fortalecer o controle social e a participação/inclusão dos usuários nos processos de planejamento (BRANDÃO; GIOVANELLA; CAMPOS, 2013).

Nesta perspectiva, o presente estudo teve por objetivo avaliar os atributos da APS com ênfase na saúde do adulto, a partir dos usuários pertencentes às áreas de abrangência das equipes de ESF de um município referência do Vale do Jequitinhonha, Minas Gerais, medidos por meio do instrumento PCATool, versão adulto.

\section{Método}

Foi realizado um estudo de delineamento transversal com uma população composta por adultos, de ambos os gêneros, com idade acima de 20 anos, cadastrados em Equipes de Saúde da Família (EqSF) de um município-polo do Vale do Jequitinhonha, Minas Gerais, em 2012. O município contava, à época do estudo, com uma população de 17.091 pessoas com idade acima de 20 anos cadastradas nas sete EqSF do referido estudo, perfazendo uma cobertura populacional de $37,2 \%$.

Assumiu-se prevalência esperada de 50\% do evento estudado, com margem de erro estimada de $4 \%$ e nível de confiança de $95 \%$. A amostra foi composta por 615 sujeitos.

Os adultos foram selecionados por meio da amostragem aleatória sistemática estratificada, a partir do cadastro dessa faixa etária no Sistema de Informação em Atenção Básica (Siab). Realizou-se, então, a distribuição de modo proporcional à real distribuição dos adultos por EqSF. Foi selecionado para a entrevista o responsável mais velho, a fim de avaliarmos a continuidade da assistência.
O intervalo amostral foi calculado dividindo-se o número total de adultos mais velhos relacionados pelo número amostral da área de abrangência. Em seguida, foram selecionados os adultos, a partir do resultado dessa divisão, aplicando-se o intervalo amostral encontrado.

Utilizou-se para a coleta de dados, o Instrumento de Avaliação da Atenção Primária (PCATool - do inglês Primary Care Assessment Tool), elaborado por Barbara Starfield e colaboradores (STARFIELD, 2002; SH; STARFIELD; XU, 2001) e validado, no Brasil, na forma de entrevista (HARZHEIM ET AL., 2006).

Os serviços referidos como preferenciais para atendimento à saúde do adulto foram definidos quando o usuário mencionou o mesmo local de atendimento para as três questões iniciais do PCATool, referentes à utilização (BRASIL, 2010A).

Foram coletados dados relativos às características demográficas e socioeconômicas. O estrato social foi definido através do Critério Padrão de Classificação Econômica Brasil, de 2005 (Critério Brasil). Os resultados obtidos na análise do perfil sociodemográfico foram expressos por frequências, por média e desvio padrão.

$\mathrm{O}$ cálculo dos escores foi realizado conforme recomendação dos organizadores do questionário PCATool - versão Adulto (BRASIL, 2010A). Após processamento dos dados, foram calculados os escores de cada atributo, do essencial (obtido através da média dos atributos essenciais e do grau de afiliação), do derivado (obtido através da média dos atributos derivados) e do geral da atenção primária (valor médio dos atributos essenciais e derivados e do grau de afiliação). Foram considerados altos escores, os valores iguais ou maiores que 6,6 , que equivalem a um ajustamento dos escores apontados na escala de Likert original a uma escala entre zero e dez, para cada um dos atributos avaliados, conforme recomendação dos estudos mais recentes que aplicam o mesmo instrumento (OLIVEIRA, 2012). 
Utilizou-se o programa estatístico Statistical Package for Social Science (SPSS) (SPSS Inc., Chicago, Estados Unidos), versão 19.0, com nível de significância $<0,05$, bem como o programa Prisma. O teste Kolmogorov-Smirnov foi utilizado para avaliar a normalidade dos dados. Devido ao fato da distribuição dos dados ser assimétrica e para avaliar os escores gerais e os escores de cada atributo com relação às áreas de abrangência, utilizou-se o teste KruskalWallis, com Post Hoc de Dunn.

O presente estudo foi aprovado pelo Comitê de Ética em Pesquisas da Universidade Federal dos Vales do Jequitinhonha e Mucuri (Protocolo 088/11).

\section{Resultados e discussão}

O município de realização do trabalho está situado na região do Vale do Jequitinhonha, Minas Gerais, Brasil, com população estimada em 45.880 habitantes (IBGE, 2010). No que tange aos serviços de APS, o município dispõe de nove equipes de ESF, havendo sete equipes na zona urbana e duas na zona rural, abrangendo $67,3 \%$ da população; e três equipes de Estratégia de Agentes Comunitários de Saúde (Eacs), situadas na zona rural, que abrangem $14,2 \%$ da população, totalizando $81,5 \%$ de cobertura populacional (DIAMANTINA, 2013).

O Instrumento de Avaliação da Atenção Primária (PCATool) utilizado neste estudo, mede a presença e a extensão dos atributos primários e derivados da atenção primária. Cada atributo é avaliado com relação a um componente de estrutura e outro de processo da atenção (HARZHEIM ET AL., 2006; BRASIL, 2010A), sendo este $\mathrm{o}$ aspecto com maior potencialidade de avaliação da qualidade (DONABEDIAN, 1984), principalmente sob a ótica do usuário - diferentemente dos demais instrumentos, como o utilizado para avaliação da qualidade da ESF, AMQ, ao utilizar uma metodologia de autoavaliação por adesão voluntária dos gestores municipais, sem considerar a avaliação na perspectiva do usuário (BRASIL, 2005), foco principal deste trabalho. Cabe ressaltar que, na fase de coleta de dados deste estudo, a gestão do município em questão não havia aderido ao AMQ e ao PMAQ (Programa de Melhoria do Acesso e da Qualidade).

De 615 indivíduos, observou-se que 64,5\% $(n=397)$ referiram que recorriam à ESF quando ficavam doentes ou precisavam de conselhos sobre a sua saúde; e 35,5\% (n=218) declararam utilizar outros serviços, entre os quais, consultório particular (28,8\%), pronto atendimento $(5,0 \%)$, ambulatórios $(1,0 \%)$ e outros serviços $(0,7 \%)$, como fonte de cuidado que não aquele ao qual estavam geograficamente adscritos (dados não demonstrados). É interessante enfatizar que, embora, $64,5 \%$ dos usuários tenham referido a ESF como provedora do cuidado, outros serviços continuam sendo procurados como fonte primária de atenção, indicando a sobreposição de rede e implantação da ESF como um programa paralelo.

No perfil sociodemográfico da população do estudo, da população que referiu procurar a $\operatorname{ESF}(n=397)$, observou-se que $71,0 \%$ $(\mathrm{n}=282)$ eram do sexo feminino, com média de idade de 50,5 anos (DP $\pm 16,1)$ (tabela 1). 


\begin{tabular}{|c|c|c|c|c|c|c|c|c|}
\hline Variáveis & $\begin{array}{l}\text { Área } 01 \\
(n=66)\end{array}$ & $\begin{array}{l}\text { Área } 02 \\
(n=57)\end{array}$ & $\begin{array}{l}\text { Área } 03 \\
(n=62)\end{array}$ & $\begin{array}{l}\text { Área } 04 \\
(n=43)\end{array}$ & $\begin{array}{l}\text { Área } 05 \\
(n=46)\end{array}$ & $\begin{array}{l}\text { Área } 06 \\
(n=53)\end{array}$ & $\begin{array}{l}\text { Área } 07 \\
(n=70)\end{array}$ & Total \\
\hline $\begin{array}{l}\text { Idade } \\
\text { (média-DP) }\end{array}$ & $\begin{array}{l}48,7 \\
( \pm 16,5)\end{array}$ & $\begin{array}{l}48,5 \\
( \pm 14,2)\end{array}$ & $\begin{array}{l}49,8 \\
( \pm 17,0)\end{array}$ & $\begin{array}{l}55,9 \\
( \pm 16,3)\end{array}$ & $\begin{array}{l}46,0 \\
( \pm 14,5)\end{array}$ & $\begin{array}{l}47,4 \\
( \pm 14,3)\end{array}$ & $\begin{array}{l}56,9 \\
( \pm 16,4)\end{array}$ & $\begin{array}{l}50,5 \\
( \pm 16,1)\end{array}$ \\
\hline \multicolumn{9}{|l|}{ Sexo } \\
\hline Masculino (\%) & 24,2 & 15,8 & 30,6 & 39,5 & 39,1 & 18,9 & 37,1 & 29,0 \\
\hline Feminino (\%) & 75,8 & 84,2 & 69,4 & 60,5 & 60,9 & 81,1 & 62,9 & 71,0 \\
\hline \multicolumn{9}{|l|}{ Escolaridade } \\
\hline $\begin{array}{l}\text { Até a } 7 \text { a série funda- } \\
\text { mental (\%) }\end{array}$ & 71,2 & 57,9 & 80,6 & 53,5 & 43,5 & 45,3 & 67,1 & 61,5 \\
\hline $\begin{array}{l}\text { Fundamental completo } \\
(\%)\end{array}$ & 10,6 & 19,3 & 3,2 & 14,0 & 21,7 & 20,8 & 1,4 & 12,1 \\
\hline Médio completo (\%) & 13,6 & 19,3 & 14,5 & 30,2 & 32,6 & 30,2 & 28,6 & 23,4 \\
\hline Superior completo (\%) & 4,5 & 3,5 & 1,6 & 2,3 & 2,2 & 3,8 & 2,9 & 3,0 \\
\hline \multicolumn{9}{|l|}{ Renda } \\
\hline $\begin{array}{l}\text { < que } 2 \text { salários míni- } \\
\text { mos (\%) }\end{array}$ & 89,4 & 89,5 & 93,5 & 72,1 & 80,4 & 73,6 & 97,1 & 86,4 \\
\hline $\begin{array}{l}\text { > que } 2 \text { salários míni- } \\
\text { mos }(\%)\end{array}$ & 10,6 & 10,5 & 6,5 & 27,9 & 19,6 & 26,4 & 2,9 & 13,6 \\
\hline \multicolumn{9}{|l|}{ Estado Civil } \\
\hline Casado (\%) & 48,5 & 33,3 & 62,9 & 60,5 & 52,2 & 51,9 & 55,7 & 51,9 \\
\hline Separado (\%) & 7,6 & 10,5 & 3,2 & 14,0 & 8,7 & 9,4 & 5,7 & 8,1 \\
\hline Viúvo (\%) & 13,6 & 22,8 & 14,5 & 14,0 & 10,9 & 11,3 & 25,7 & 16,6 \\
\hline $\begin{array}{l}\text { Solteiro/não informado } \\
\text { (\%) }\end{array}$ & 30,3 & 33,3 & 19,4 & 11,6 & 28,3 & 28,3 & 12,9 & 23,4 \\
\hline \multicolumn{9}{|l|}{ Plano de saúde } \\
\hline $\operatorname{Sim}(\%)$ & 13,6 & 8,8 & 3,2 & 20,9 & 6,5 & 20,8 & 11,4 & 11,8 \\
\hline Não (\%) & 86,4 & 91,2 & 96,8 & 79,1 & 93,5 & 79,2 & 88,6 & 88,2 \\
\hline
\end{tabular}

Nota: Elaboração própria

Essa maior demanda feminina para participar da pesquisa acompanha o perfil da transição demográfica brasileira, principalmente em áreas urbanas, que se caracteriza por uma maior concentração de mulheres, com especial ênfase nas idades mais avançadas (PINHEIRO, 2002). 86,4\% ( $\mathrm{n}=343)$ dos entrevistados declararam renda média menor que dois salários mínimos e baixa escolaridade, sendo $61,5 \%(n=244)$ com até o ensino fundamental. $51,9 \%(\mathrm{n}=206)$ eram casados, $16,6 \%(n=66)$ eram viúvos (tabela 1). O perfil sociodemográfico dos indivíduos que utilizavam a ESF apontou vulnerabilidade econômica e social. Esses resultados se assemelham a outro trabalho em que os estratos mais pobres da população utilizavam mais os serviços da ESF como referência para a assistência (SIQUEIRA, 2007). Foi observado que $88,2 \%(n=350)$ relataram não possuir plano de saúde (tabela 1). Este dado aproxima-se de outro estudo (SILVA, 2011), em que mais de $80 \%$ dos usuários dos serviços de APS não possuíam planos de saúde, confirmando que o SUS é o maior agente de financiamento da atenção à saúde do País.

A média do escore geral para avaliação da APS do município, obtido pelas médias dos escores dos atributos de cada área, resultou em um valor de 4,5 (gráfico 1), abaixo do escore considerado adequado pela literatura (alto escore: $\geq 6,6$ ), segundo Harzheim et al. (2006). 
Gráfico 1. Escore geral (EG) por área de abrangência do município, 2012

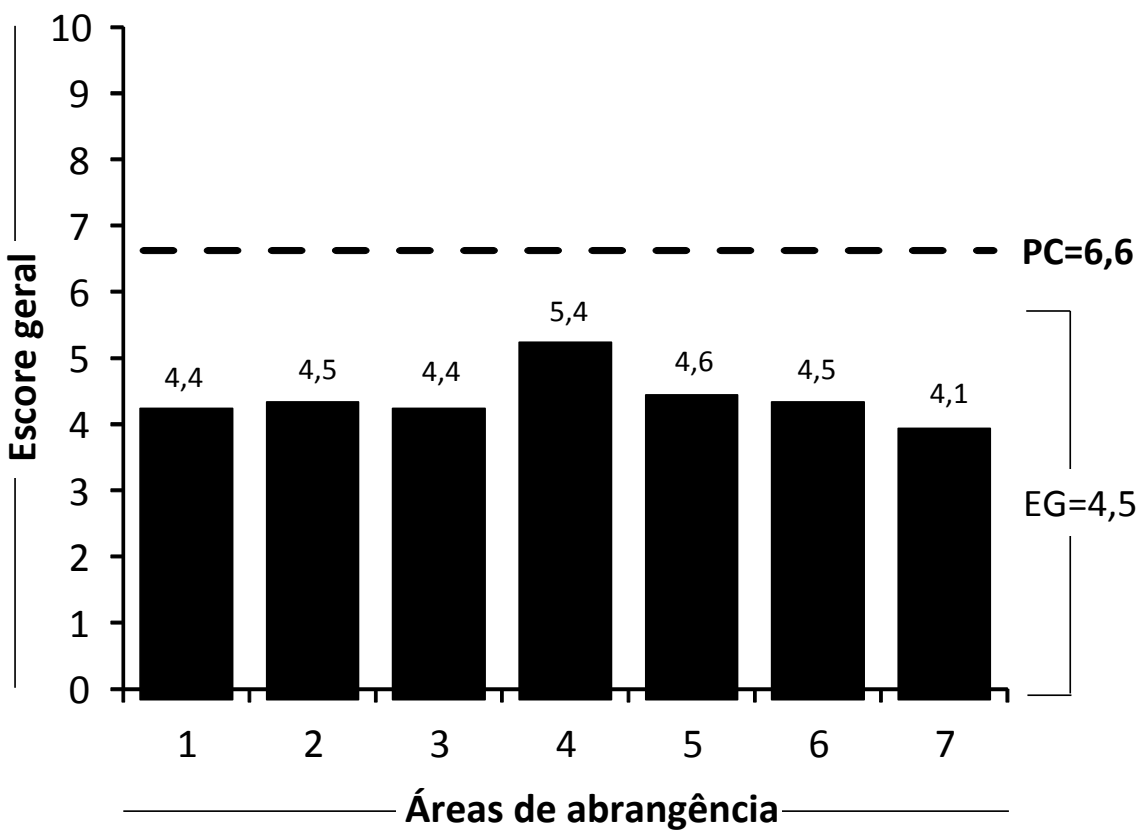

Notas: Elaboração própria

Cada barra representa o escore geral obtido por área de abrangência do município. Escore geral $(E G)=4,5$. $O$ ponto de corte $(P C=6,6)$ está representado pela linha tracejada ao longo do gráfico

Percebeu-se que os serviços de saúde escolhidos pelos usuários como fonte regular de atenção à saúde do adulto não estão fornecendo atenção em consonância com os atributos essenciais e derivados da APS. Dessa forma, sendo a ESF um modelo de reorganização e fortalecimento da APS (STARFIELD, 2002), esses achados indicam a necessidade de melhoria do serviço, mediante reformulação do processo de trabalho por parte dos gestores e profissionais de saúde para consolidação da sua efetividade.
Cabe ressaltar, entretanto, que o escore geral obtido para a área 04 (escore $=5,4)$ mostrou-se superior ao escore geral obtido para as outras áreas (área 01, escore $=4,4$; área 02, escore $=4,5$; área 03, escore $=4,4$; área 05, escore $=4,6$; área 06, escore $=4,5 \mathrm{e}$ área 07 , escore $=4,1$ ) (gráfico 1). Para melhor compreensão do escore geral observado em cada área estudada, foi identificada a média dos escores de cada atributo de todas as áreas de abrangência (gráfico 2). 
Gráfico 2. Escore médio (EM) por atributo da Atenção Básica, 2012

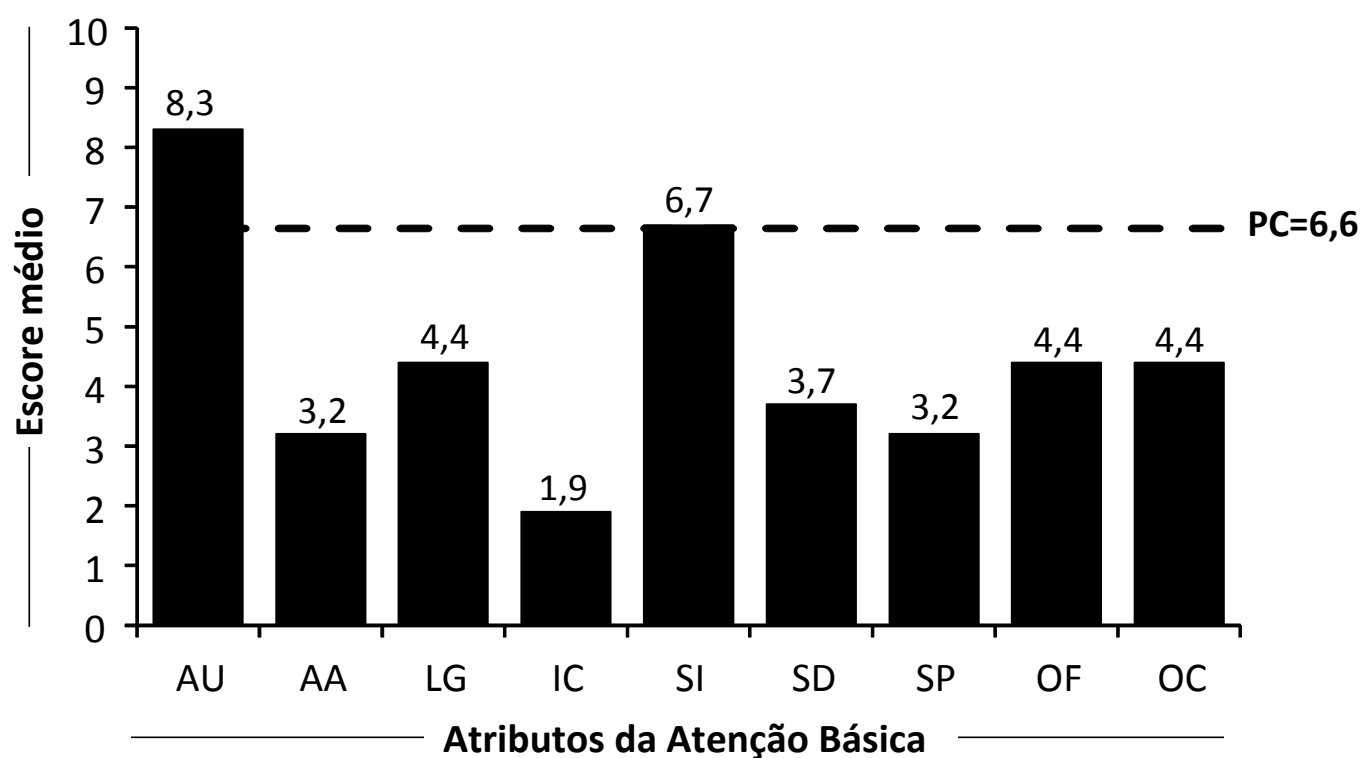

Notas: Elaboração própria

Cada barra representa o escore médio por atributo da Atenção Básica ( $\mathrm{AU}=$ Acesso de primeiro contato - utilização; $\mathrm{AA}=\mathrm{Acesso}$ de primeiro contato - acessibilidade; $L G=$ Longitudinalidade; $I C=$ Integração dos cuidados; $S I=$ Sistemas de informação; SD = Serviços disponíveis; SP = Serviços prestados; OF = Orientação familiar; e OC = Orientação comunitária) do município. O ponto de corte (PC=6,6) está representado pela linha tracejada ao longo do gráfico

Verificou-se que apenas os atributos 'Acesso de primeiro contato - Utilização’ e 'Sistemas de informação' apresentaram alto escore (acima do ponto de corte 6,6): 8,3 e 6,7, respectivamente. Todos os outros atributos apresentaram baixo escore (abaixo do ponto de corte 6,6), sendo, 3,2 para os atributos 'Acesso de primeiro contato - Acessibilidade' e 'Serviços prestados', 3,7 para o atributo serviços disponíveis, 6,3 para o atributo 'Longitudinalidade', 4,4 para os atributos 'Orientação familiar' e 'Orientação comunitária' e, com a menor pontuação, 1,9 para o atributo 'Integração de cuidados' (gráfico 2).
Para aprofundar a avaliação da APS de cada área de abrangência do município em estudo, com o intuito de compreender os resultados demonstrados no gráfico 2, foi utilizada a média dos valores obtidos para cada atributo, de acordo com orientações contidas no Manual do Instrumento de Avaliação da Atenção Primária à Saúde - PCATooL (BRASIL, 2010A), apresentados em gráfico de radar (figura 1, gráficos à esquerda). Entretanto, por se tratar de escores individuais que apresentaram distribuição não paramétrica para todos os atributos, utilizaram-se medianas para a análise estatística dos dados (figura 1, gráficos à direita). 
Figura 1. Avaliação dos atributos da Atenção Básica de cada área de abrangência do município, 2012

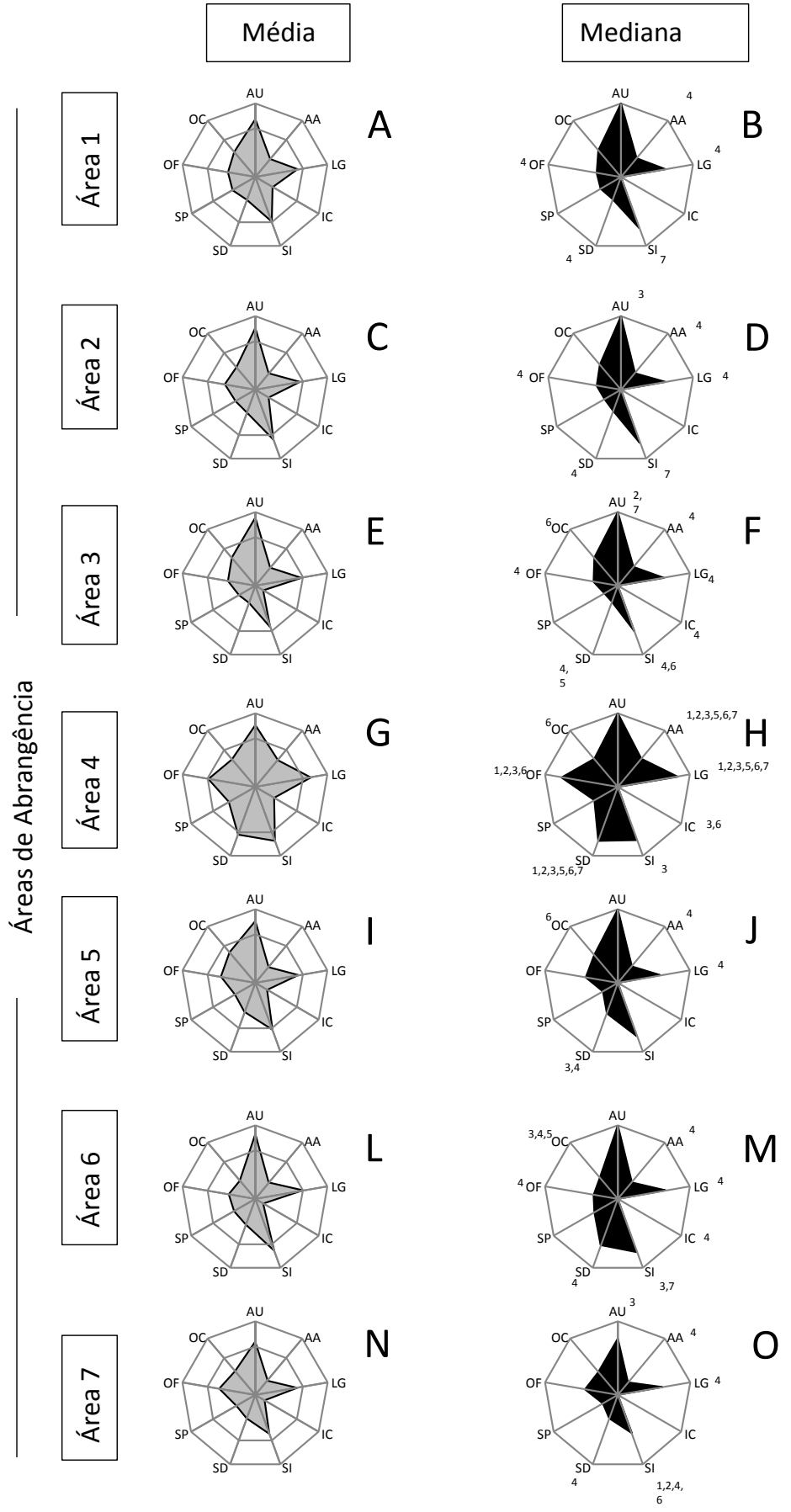

Notas: Elaboração própria

Gráficos de radar à esquerda: distribuição dos escores médios dos atributos da Atenção Básica (AU = Acesso de primeiro contato utilização; $A A$ = Acesso de primeiro contato - acessibilidade; $L G$ = Longitudinalidade; $I C=$ Integração dos cuidados; $S I$ = Sistemas de informação; $\mathrm{SD}$ = Serviços disponíveis; $\mathrm{SP}$ = Serviços prestados; $\mathrm{OF}=$ Orientação familiar; e OC = Orientação comunitária) por área de abrangência do município (escala de 0 a 10 e ponto de corte $(P C=6,6)$ representado pela linha no centro do radar. Gráficos de radar à direita: comparações múltiplas das medianas dos atributos da Atenção Básica entre as áreas de abrangência do município. Utilizou-se Teste Kruskal-Wallis, com Post Hoc de Dunn $(p<0,05)$. Diferenças estatísticas entre as áreas são representadas pelos números sobrescritos às siglas de cada atributo 
$\mathrm{Na}$ análise do atributo 'Acesso de primeiro contato - Utilização’ (AU), a avaliação foi favorável em todas as áreas de abrangência (figura 1, gráficos à esquerda). Pôde-se perceber, também, um padrão mais uniforme na avaliação: a área 03 diferiu significativamente apenas das áreas 02 e 07 (figura 1, gráfico F). A avaliação favorável e homogênea desse atributo já era esperada, levando-se em conta a população estudada (usuários que referiram a ESF como responsável pelo seu cuidado, $\mathrm{n}=397$ ). Embora o estudo apresente essa limitação, outro estudo já identificou um bom desempenho da ESF como porta de entrada para o serviço de saúde (GIOVANELLA ET AL., 2009).

Adicionalmente, apesar de todas as áreas de abrangência das EqSF terem ficado aquém do nível satisfatório para o atributo 'Acesso de primeiro contato - Acessibilidade' (AA) (figura 1, gráficos A, C, E, I, L, N), a área de abrangência 04 apresentou melhor projeção (escore $=5,0$ ) (figura 1, gráfico $\mathrm{G}$ ) e diferiu estatisticamente de todas as outras áreas avaliadas (figura 1, gráfico H). Pode-se inferir que a área 04 esteja sendo contemplada com melhor oferta de serviços por parte da equipe responsável pela população adscrita, sem que haja maiores barreiras geográficas restritivas ao seu acesso. Entretanto, os resultados encontrados para as outras áreas do município indicaram que esse atributo é um desafio a ser enfrentado. $\mathrm{O}$ achado mostrou-se semelhante a estudo realizado no município de Colombo (PR) (OLIVEIRA, 2012), que utilizou PCATool infantil para avaliação dos atributos da APS.

Na avaliação do atributo 'Longitudinalidade' (LG), foi observado baixo escore $(\leq 6,6)$ em cinco áreas (figura 1, gráficos A, C, E, I e N), exceto nas áreas 04 e 06 (figura 1, gráficos $\mathrm{G}$ e L). Esse atributo é considerado característica central da APS, segundo Braz (2012), e está fortemente relacionado à boa comunicação, que tende a favorecer o acompanhamento do paciente, a continuidade e a efetividade do tratamento, contribuindo para a implementação de ações de promoção e de prevenção de agravos de alta prevalência. Apesar desse resultado geral não satisfatório, a área de abrangência 04 apresentou desempenho elevado (escore $=8,3$ ), com diferença estatisticamente significativa em relação às demais áreas (figura 1, gráfico $\mathrm{H})$. Contrariamente, em estudo conduzido por Silva et al. (2014), em município-polo do Norte de Minas Gerais, o atributo longitudinalidade apresentou-se com percentual significativamente maior de avaliações de alto escore entre os usuários da ESF (80\%).

$\mathrm{Na}$ avaliação do atributo 'Integração de Cuidados' (IC), os valores ficaram muito aquém do escore desejável em todas as áreas avaliadas (figura 1, gráficos à esquerda). Resultado semelhante foi encontrado em Sobral (CE), em que, na opinião dos usuários das 15 unidades de saúde da família, 11 apresentaram baixo escore para esse atributo (CARNEIRO ET AL., 2014). Esses achados sugerem a ausência de um sistema organizado, com fragmentação da atenção nos diferentes níveis locais de prestação de serviços, e reforça a falta de uma sólida informação de base, bem como a necessidade de estratégias sistemáticas para desenvolvê-la (STARFIELD, 2002). Assim, potencializar a APS como centro ordenadora e integradora das redes de serviços e das ações de promoção, prevenção e recuperação da saúde é um dos principais desafios segundo os gestores $\mathrm{mu}$ nicipais (GIOVANELLA ET AL., 2009).

$\mathrm{Na}$ avaliação do atributo 'Sistema de informações' (SI), houve avaliação favorável em quatro áreas (02, 04, 05, 06) (figura 1, gráficos C, G, I e L, respectivamente) e desfavorável em três áreas (01, 03 e 07) (figura 1, gráficos A, E e N, respectivamente). Menores valores de mediana foram observados nas áreas 03 e 07 (6,6 e 5,5 respectivamente) (figura 1, gráficos $\mathrm{F}$ e $\mathrm{O}$, respectivamente). Diferenças estatísticas também foram verificadas: área 07 difere das áreas 01, 02, 04 e 06 (figura 1, gráfico O); e área 03 difere das áreas 04 e 06 (figura 1, gráfico F). Em estudo de Carneiro et al. (2014), foi observado que, em um total de 15 unidades avaliadas, apenas uma obteve escore abaixo 
da média para esse atributo. Tais achados sugerem que os profissionais e usuários de algumas áreas possuem adequado acesso aos registros de atendimentos em prontuários, condição importante para um bom acompanhamento de usuários e famílias pelos serviços de saúde, enquanto outras, não.

$\mathrm{Na}$ análise do atributo 'Serviços Disponíveis' (SD), somente a área 04 atingiu escore favorável $(7,8)$ (figura 1 , gráfico $\mathrm{G}$ ) e se apresentou diferente, estatisticamente, de todas as outras áreas (figura 1, gráfico $\mathrm{H})$. No tocante às dimensões da integralidade 'Serviços Disponíveis', observaram-se valores do escore aquém do ideal também no estudo de Braz (2012). Na avaliação do atributo 'Serviços prestados' (SP), todas as áreas apresentaram médias abaixo do ponto de corte (figura 1, gráficos à esquerda), e não foram demonstradas diferenças significativas entre as EqSF avaliadas (figura 1, gráficos à direita). A avaliação negativa do atributo 'Integralidade' sugere que nem todas as ações de promoção e prevenção da saúde, voltadas ao cuidado integral ao adulto estão sendo executadas de forma efetiva, exceto na área 04. Contrariamente, em estudo utilizando inquéritos do PMAQ-AB (Programa Nacional de Melhoria do Acesso e da Qualidade da Atenção Básica), $73 \%$ dos usuários consideram que a equipe busca resolver suas necessidades na própria unidade, avaliando positivamente a integralidade (FAUSTO ET AL., 2014).

Com relação à avaliação do atributo 'Orientação Familiar' (OF), todas as áreas apresentaram médias abaixo do ponto de corte, (figura 1, gráficos à esquerda), achado que reflete o quanto a família não tem sido destaque no processo de formação e de assistência à saúde nas unidades de EqSF municipais. Em outro estudo, realizado por Pimenta et al. (2008), em uma ESF de Amparo, São Paulo, utilizando o PCATool, o atributo 'orientação familiar' apresentou, na experiência dos usuários, o escore mais baixo. Oliveira (2012, P. 91) cita em seu trabalho uma afirmativa feita por Freitas (2008), de que embora a ESF desde a sua implantação apresente como foco a família, não se evidência, nos documentos analisados e especialmente na Política Nacional de Atenção Básica (PNAB), nas proposições tecnológicas e finalidades a valorização da família e de suas redes de relações.

Os resultados encontrados na área de abrangência 04 mostraram diferenças estatísticas de outras quatro áreas (áreas 01, 02, 03 e 06), apresentando-se com melhor orientação para esse atributo (figura 1, gráfico $\mathrm{H}$ ), o que sugere que essa área focaliza a família de forma diferenciada.

A respeito do atributo 'Orientação Comunitária' (OC), todas as áreas apresentaram médias abaixo do ponto de corte (figura 1, gráficos à esquerda). Cabe enfatizar que a área 04 apresentou escore médio muito próximo ao ponto de corte (escore $=6,5$ ) (figura 1, gráfico G). Na avaliação comparativa entre as áreas, constataram-se diferenças estatísticas negativas da área 06 em relação a três outras áreas (áreas 03, 04 e 05), pois, a área 06 apresentou o pior desempenho para tal atributo, com valor de mediana igual a 3,8 (figura 1, gráfico M). Tal resultado evidencia que a atenção à saúde desenvolvida pelas EqSF não está focalizando corretamente a comunidade na qual estão inseridas. Resultados semelhantes foram encontrados no estudo de Braz (2012) e Alencar et al. (2014), em que o atributo não teve sequer uma avaliação satisfatória pelos usuários.

Verificaram-se diferenças pertinentes entre as diversas áreas que compõem o município, o que aponta uma necessidade de sistematização das ações e serviços. Uma provável explicação para a heterogeneidade dos resultados apresentados por cada área de abrangência, com relação à operacionalização dos atributos da APS, pode estar associada a diferentes estruturas, tanto físicas quanto organizacionais, das equipes responsáveis por tais áreas, além de influência de determinantes sociais, comprometendo todo um processo de trabalho e, consequentemente, o processo avaliativo. 
É importante salientar que o método de avaliação dos serviços proposto pelo PCATool é muito eficaz, porém, é necessário avançar nas investigações de estrutura, processo de trabalho e determinação social.

As limitações do estudo ocorreram em consequência do instrumento PCATool. A primeira é considerar, para o cálculo dos Escores Essencial e Geral, que os atributos tenham os mesmos 'pesos' na medida da orientação à APS. A segunda reside em utilizar apenas a experiência dos atores envolvidos na atenção como critério avaliador, não incorporando, por exemplo, a avaliação técnica do serviço prestado.

Além disso, outras limitações são aquelas inerentes à pesquisa avaliativa, transversal, sujeita às limitações próprias desse tipo de delineamento. Uma delas é a validade externa, já que os resultados da pesquisa avaliativa de serviços devem ser discutidos considerando-se a realidade local dos serviços.

\section{Conclusão}

Os componentes avaliados permitiram apontar que alguns atributos da APS não estão sendo efetivados no que se refere à assistência integral à saúde do adulto no município estudado. Os achados evidenciaram uma ineficiência do serviço em prover as necessidades de saúde da população adulta. O cuidado integral, não se encontra articulado às reais necessidades dos indivíduos, das famílias e comunidades, não sendo capaz de contribuir para a ampliação do conceito de saúde e/ou do viver saudável, além de não possibilitar elementos para a consolidação da ESF.

Os resultados apresentados neste estudo trazem importantes implicações para os serviços avaliados e também para as políticas públicas de atenção à saúde do adulto. Por isso, espera-se que a divulgação dessas evidências aos gestores e profissionais de saúde possibilite e gere discussões, transformações e reorganização de suas práticas, possibilitando uma maior inserção da população na formulação das atividades, rumo à reformulação do sistema de saúde hegemônico vigente.

Acredita-se que este trabalho poderá subsidiar futuras práticas e decisões acerca do investimento público, visando ao fortalecimento da Atenção Básica pelos gestores municipais. 


\section{Referências}

ALENCAR, M. N. et al. Avaliação do enfoque familiar e orientação para a comunidade na Estratégia Saúde da Família. Ciência \& Saúde Coletiva, Rio de Janeiro, v. 19, n. 2, p. 353-364, 2014.

BRASIL. Ministério da Saúde, Secretaria de Atenção à Saúde, Departamento de Atenção Básica. Diabetes Mellitus. Brasília, DF: Ministério da Saúde, 2006.

Ministério da Saúde, Secretaria de Atenção em Saúde, Departamento de Atenção Básica. Manual do instrumento de avaliação da Atenção Primária à Saúde: Primary Care Assessment Tool Pcatool - Brasil. Brasília, DF: Ministério da Saúde, 2010a.

Ministério da Saúde. O que é $A M Q$ ? Brasília,DF: Ministério da Saúde, 2010b. Disponível em: $<$ http://189.28.128.100/dab/proesf/docs/geral/4-OficinaSGP2-AMQ.pdf>. Acesso: 15 jun. 2013.

Ministério da Saúde. Secretaria da Atenção à Saúde, Departamento de Atenção Básica. Avaliação na atenção básica em saúde. Brasília, DF: Ministério da Saúde, 2005a.

Ministério da Saúde. Secretaria de Atenção à Saúde. Departamento de Atenção Básica. Política Nacional de Atenção Básica. 4 ed. Brasília, DF: Ministério da Saúde, 2007.

BRANDÃO, A. L. R. B. S.; GIOVANELLA, L.; CAMPOS, C. E. A. Avaliação da atenção básica pela perspectiva dos usuários: adaptação do instrumento EUROPEP para grandes centros urbanos brasileiros. Ciência \& Saúde Coletiva, Rio de Janeiro, v. 18, n. 1, p. 103-114, 2013.

BRAZ, J. C. Avaliação da atenção às crianças menores de um ano nas Estratégias de Saúde da Família em um município da Bahia, sob a ótica dos cuidadores. 2012. 86 f. Dissertação (Mestrado em Enfermagem) Universidade de São Paulo, Escola de Enfermagem de Ribeirão Preto, Ribeirão Preto, 2012.

CARNEIRO, M. S. M. et al. Avaliação do atributo coordenação da Atenção Primária à Saúde: aplicação do PCATool a profissionais e usuários. Saúde em Debate, Rio de Janeiro, v. 38, n. especial, p. 279-295, out. 2014.
DIAMANTINA (Cidade). Secretaria municipal de Diamantina, 2013. Disponível em: $<$ http://www.diamantina.mg.gov.br>. Acesso em: 15 jun. 2013.

FAUSTO, M. C. R. et al. A posição da Estratégia Saúde da Família na rede de atenção à saúde na perspectiva das equipes e usuários participantes do PMAQ-AB. Saúde em Debate, Rio de Janeiro, v. 38, n. especial, p. 13-33, out. 2014.

FIGUEIREDO, A. M. et al. Análise de concordância entre instrumentos de avaliação da Atenção Primária à Saúde. Epidemiol. Serv. Saúde, Brasília, DF, v. 22, n. 1, p. 41-48, 2013.

FREITAS, M. L. A. Referências de qualidade relativas ao programa de saúde da família: um estudo em documentos da política de saúde nacional. 2008. $233 \mathrm{f}$. Dissertação (Mestrado em Enfermagem) - Faculdade de Enfermagem, Universidade Federal do Mato Grosso, Cuiabá, 2008.

GIOVANELLA, L. et al. Saúde da família: limites e possibilidades para uma abordagem integral de atenção primária à saúde no Brasil. Ciência \&t Saúde Coletiva, Rio de Janeiro, v. 14, n. 3, p. 783-794, 2009.

HARZHEIM, E. et al. Revisão sistemática sobre aspectos metodológicos das pesquisas em atenção primária no Brasil. Revista da AMRIGS, Porto Alegre, v. 49, n. 4, p. 248-252, 2005.

HARZHEIM, E. et al. Internal consistency and reliability of Primary Care Assessment Tool (PCATool-Brasil) for child health services. Cadernos de Saúde Pública, Rio de Janeiro, v. 22, n. 8, p. 1649-1659, 2006.

HARZHEIM, E. et al. Reforma da Atenção Primária à Saúde na cidade do Rio de Janeiro - avaliação dos três anos de Clínicas da Família. Pesquisa avaliativa sobre aspectos de implantação, estrutura, processo e resultados das Clínicas da Família na cidade do Rio de Janeiro. Porto Alegre: OPAS, 2013.

INSTITUTO BRASILEIRO DE GEOGRAFIA E ESTATÍSITICA (IBGE). Cidades. Disponível em: $<$ http://www.ibge.gov.br/cidadesat/topwindow.htm?1>. Acesso: 1 mar. 2013. 
OLIVEIRA, V. B. C. A. Avaliação da Atenção Primária à Saúde da criança no município de Colombo - PR. 2012. 118 f. Dissertação (Mestrado em Enfermagem) Universidade de São Paulo, Escola de Enfermagem de Ribeirão Preto. Ribeirão Preto, 2012.

SILVA, C. S. O. et al. Integralidade e Atenção Primáriaà Saúde: avaliação sob a ótica dos usuários. Ciência \&t Saúde Coletiva, Rio de Janeiro, v. 19, n. 11, p. 4407-4415, 2014.

PASARÍN, M. I. An instrument to evaluate primary health care from the population perspective. Aten Primaria, Barcelona, v. 39, n. 8, p. 395-401, 2007.

PIMENTA, A. et al. Pesquisa sobre organização e desempenho das Unidades de Saúde da Família de Amparo (SP): utilização de metodologia de avaliação rápida. Saúde em Debate, Rio de Janeiro, n. 42, p. 102-17, 2008.

PINHEIRO, R. S. et al. Gênero, morbidade, acesso e utilização de serviços de saúde no Brasil. Ciência \& Saúde Coletiva, Rio de Janeiro, v. 7, n. 4, p. 687-707, 2002.

RIBEIRO, L. C. C. Acolhimento às crianças na atenção primária à saúde: um estudo sobre a postura dos profissionais das equipes de saúde da família. 2009. 105 f. Dissertação
(Mestrado em Ciências da Saúde) - Universidade Federal de Minas Gerais, Belo Horizonte, 2009.

SHI, L.; STARFIELD, B.; XU, J. Validating the adult primary care assessment tool. The Journal of Family Practice, Oxford, v. 50, n. 2, p. 161-75, 2001.

SILVA, Z. P. et al. Perfil sociodemográfico e padrão de utilização dos serviços de saúde do Sistema Único de Saúde (SUS), 2003-2008. Ciência \&t Saúde Coletiva, Rio de Janeiro, v. 16, n. 9, p. 3807-3816, 2011.

SIQUEIRA, B. R. Miséria. 3 ed. Rio de Janeiro: KroArts, 2007.

STARFIELD, B. Primary care: concept, evaluation and policy. New York: Oxford University Press, 1992.

STARFIELD, B. Atenção Primária: equilíbrio entre as necessidades de saúde, serviços e tecnologia. Brasília, DF: UNESCO: Ministério da Saúde, 2002.

Recebido para publicação em abril de 2014

Versão final em janeiro de 2015

Conflito de interesses: inexistente

Suporte financeiro: não houve 